\title{
The Effect of Concomitant Arthroscopic Lateral Retinacular Release on Postoperative Patellar Position and Orientation in Open Wedge High Tibial Osteotomy
}

Kazuhiro Murayama, $\mathrm{MD}^{1}$, Hiroshi Nakayama, $\mathrm{MD}^{1}$, Tomohiko Murakami, $\mathrm{MD}^{2}$, Shinichi Yoshiya, $\mathrm{MD}^{1}$, Shuhei Otsuki, $\mathrm{MD}^{2}$, and Toshiya Tachibana, $\mathrm{MD}^{1}$

${ }^{1}$ Department of Orthopaedic Surgery, Hyogo College of Medicine, Nishinomiya; ${ }^{2}$ Department of Orthopedic Surgery, Osaka Medical College, Osaka, Japan

Purpose: The purpose of this study was to evaluate the effect of concomitant arthroscopic lateral release (LR) in open wedge high tibial osteotomy (OWHTO) by comparing the pre- and postoperative radiological parameters of patellar position and orientation.

Materials and Methods: The study was comprised of 19 knees undergoing OWHTO and concomitant LR and 18 knees undergoing OWHTO alone. Radiological parameters for patellar position and orientation included the Caton-Deschamps index (CDI), Blackburne-Peel index (BPI), patellar tilting angle (PTA), patellar lateral shift (PLS), and patellofemoral distance (PFD), which were evaluated in the preoperative period and at one year after surgery.

Results: Patellar height was significantly reduced after surgery as indicated by the decrease in BPI ( $\mathrm{p}=0.03$ ) in the OWHTO/LR group, and decrease in CDI ( $\mathrm{p}=0.03)$ and BPI $(\mathrm{p}=0.04)$ in the OWHTO alone group. PTA and PLS were significantly reduced after the combined OWHTO/LR procedure $(\mathrm{p}=0.04$ and $\mathrm{p}=0.04$, respectively). By contrast, no significant changes were detected when isolated OWHTO was performed.

Conclusions: OWHTO induced a postoperative decrease in patellar height in both groups. Regarding the change in patellofemoral alignment, concomitant LR in OWHTO significantly decreased lateral patellar tilt and shift, while no significant difference in those parameters were noted in the OWHTO alone knees.

Keywords: Knee, Osteotomy, Open wedge, Arthroscopy, Lateral retinacular release, Patellar position

\section{Introduction}

Open wedge high tibial osteotomy (OWHTO) is a commonly performed procedure for knees exhibiting medial compartment osteoarthritis with varus malalignment ${ }^{1}$, and favorable surgical outcomes have been reported in the majority of clinical follow-up studies ${ }^{2,3}$. However, this procedure also carries the risk of com-

Received January 13, 2018; Revised (1st) April 19, 2018;

(2nd) July 9, 2018; Accepted July 23, 2018

Correspondence to: Kazuhiro Murayama, MD

Department of Orthopaedic Surgery, Hyogo College of Medicine, 1-1

Mukogawa-cho, Nishinomiya, Hyogo 663-8501, Japan

Tel: +81-798-45-6452, Fax: +81-798-45-6453

E-mail: studiokaz@msn.com

This is an Open Access article distributed under the terms of the Creative Commons Attribution Non-Commercial License (http://creativecommons.org/licenses/by-nc/4.0/) which permits unrestricted non-commercial use, distribution, and reproduction in any medium, provided the original work is properly cited. plications such as nonunion, infection, tibial plateau fracture and patellofemoral problems ${ }^{4-8)}$.

Among these complications, postoperative patellofemoral problems such as patella baja and anterior knee pain have been considered as potential shortcomings of OWHTO because this procedure inevitably lowers the tibial tubercle position creating a potential risk for patella baja and increases patellofemoral pressure $^{2,4,9-12)}$. Therefore, measures against these unfavorable patellofemoral mechanics are essential to improve the outcomes of OWHTO.

Lateral retinacular release is a popular arthroscopic procedure performed to improve the patellofemoral alignment and relieve the excessive joint pressure ${ }^{8}$. Therefore, concomitant lateral release (LR) at the time of OWHTO may effectively improve patellofemoral mechanics leading to reduced anterior knee symptoms and improved surgical outcome and patient satisfaction.

There have been a few studies that examined the effect of concomitant LR on the postoperative patellofemoral mechanics and 
outcomes of high tibial osteotomy (HTO). Christodoulou et al. ${ }^{9)}$ compared the results of HTO with and without LR and reported that concomitant LR could afford better postoperative patellofemoral alignment, range of motion, and clinical score compared to isolated HTO without LR. However, their HTO procedure was a Coventry-type closed wedge osteotomy. Saito et al. ${ }^{13)}$ reported that anterior advancement of distal fragment associated with LR could improve patellofemoral alignment; however, their series also dealt with subjects with LR combined with closed wedge HTO. To date, the efficacy of LR for the improvement of patellofemoral mechanics in OWHTO has not been reported.

Therefore, the primary purpose of this study was to evaluate the effect of concomitant arthroscopic LR in OWHTO on postoperative patellar position and orientation based on the comparison of pre- and postoperative radiological parameters. It was hypothesized that concomitant LR would improve the postoperative patellofemoral mechanics as evidenced by radiological findings.

\section{Materials and Methods}

The subjects of this study were composed of a consecutive series of 19 knees undergoing OWHTO concomitant with LR (OWHTO/LR group) and 18 knees undergoing OWHTO alone without LR (OWHTO alone group). The patients and/or their families were informed that data from the case would be submitted for publication and gave their consent. The surgeries were performed by two surgeons ( $\mathrm{HN}$ and $\mathrm{SO}$ ). During the period from January 2013 to March 2014, one surgeon (HN) performed OWHTO concomitant with LR in all cases when the opening wedge width was more than $10 \mathrm{~mm}$, while the other surgeon (SO) performed isolated HTO for all knees regardless of the wedge width.

A

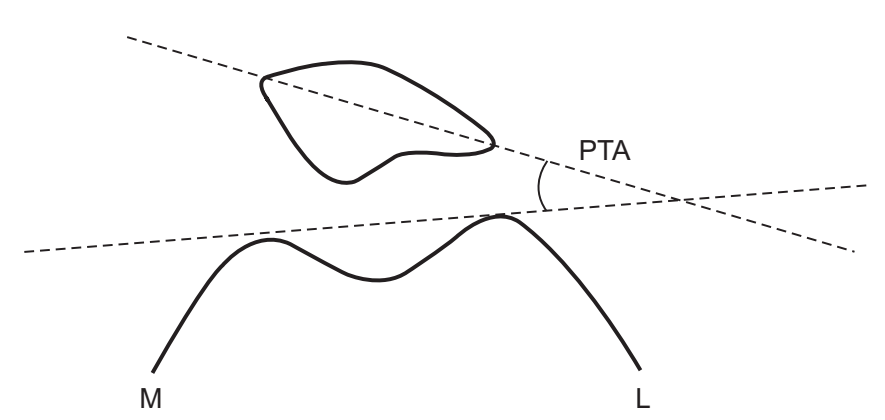

All knees underwent OWHTO for medial compartment osteoarthritis with varus deformity after failure of supervised conservative treatment. OWHTO was not indicated for knees with substantial patellofemoral osteoarthritis and symptoms, rheumatoid arthritis, flexion contracture of $>10^{\circ}$, and total range of motion $<120^{\circ}$. In order to make a comparison between knees undergoing OWHTO with comparable amount of correction, knees with a wedge width of less than $10 \mathrm{~mm}$ were excluded from the study population. In addition, knees with other concomitant procedures such as anterior cruciate ligament reconstruction were also excluded.

At surgery, the lateral patellar retinaculum was released with a radiofrequency device under arthroscopic view. The release area was between the anterolateral portal and the proximal pole of the patella as in other studies reported in the literature ${ }^{14,15)}$.

A TomoFix plate (DePuy Synthes, Zuchwil, Switzerland) was used to attain solid fixation at the osteotomy site, and a $\beta$ TCP spacer was inserted into the open gap.

Postoperatively, the same rehabilitation protocol was followed in both groups. Patients were allowed range of motion and straight leg raising exercises from the 1st postoperative day. Touchdown weight bearing was started at 1 week after surgery with full weight bearing allowed at 2 weeks after surgery.

Radiological examination included a bilateral standing anteroposterior whole leg radiograph, standing anteroposterior and lateral views, and an axial view. The radiographs obtained preoperatively and at 1 year after surgery were used for the analysis, and pre- and postoperative results were compared. Examined radiological parameters were as follows: coronal limb alignment (anatomical femorotibial angle), posterior slope of the proximal tibia (tibial slope) ${ }^{16)}$, Caton-Deschamps index (CDI) and

B

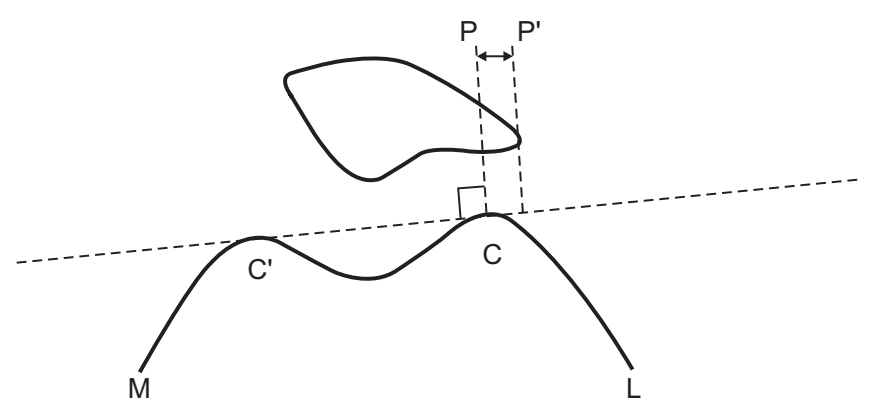

Fig. 1. (A) Measurement of patellar tilting angle (PTA). PTA was defined as the angle between a line intersecting the widest bony structure of the patella and a line tangent to the anterior surface of the femoral condyles on a skyline view. (B) Measurement of patellar lateral shift (PLS). PLS was defined as the ratio of PP' to CC.' CC': distance between the summits of the condyles on the medial and lateral sides, PP': distance between the summit of the lateral condyle and the point where a line drawn from the lateral edge of the patella runs perpendicular to a line that passes through the summits of lateral (L) and medial (M) condyles. 
Blackburne-Peel index (BPI) on the lateral radiograph to assess the patellar height in reference to the joint level ${ }^{13)}$, patellar tilting angle (PTA) and patellar lateral shift (PLS) (Fig. 1) ${ }^{2}$, and medial and lateral patellofemoral joint distances (M-PFD and L-PFD) (Fig. 2) as parameters for patellofemoral alignment measured on the $30^{\circ}$ axial view ${ }^{2)}$.

Mann-Whitney $U$-test was used to statistically analyze the difference in numerical variables between the groups as well as preand postoperative radiological results. The difference in gender distribution between the groups was assessed using the Fisher exact test.

This study was performed after obtaining Institutional Review Board approval from our institution.

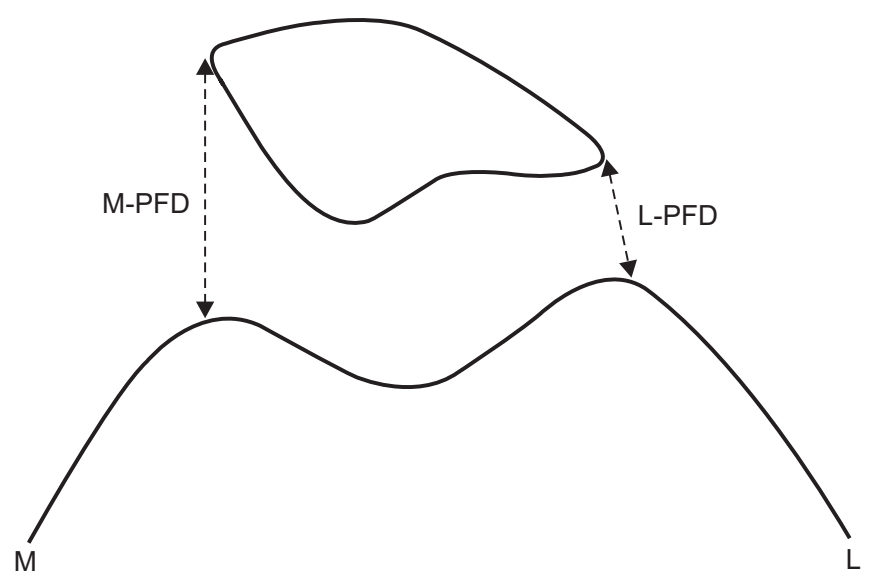

Fig. 2. Measurement of patellofemoral distance (PFD). Distance from the lateral (medial) edge of the lateral trochlea to the lateral (medial) edge of the patella was measured on a skyline view and defined as lateral PFD (L-PFD) and medial PFD (M-PFD), respectively. M: medial, L: lateral.

\section{Results}

Preoperative demographic and clinical characteristics in both groups are comparatively shown in Table 1. All demographic and clinical characteristics were comparable without significant intergroup difference except for age (52.0 years and 63.1 years on average for the OWHTO/LR group and OWHTO alone group, respectively).

The mean opening height was $12.8 \mathrm{~mm}$ and $11.8 \mathrm{~mm}$ in the OWHTO/LR and OWHTO alone groups, respectively. Regarding intra- and postoperative complications, there was no intraoperative fracture on the lateral side of tibia requiring additional procedure or modification of the postoperative protocol. Delayed union at the osteotomy site was not encountered in the study subjects. One patient in the OWHTO/LR group sustained superficial wound infection which was resolved by local management.

Pre- and postoperative values measured for each of the radiological parameters in both groups are comparatively shown in Table 2. In the OWHTO/LR group, patellar height significantly decreased in the assessment of BPI, while a significant postoperative reduction in CDI was detected in the OWHTO alone group. Regarding patellar shift and tilt, in the OWHTO/LR group, both PTA and PLS values significantly decreased after surgery indicating significant postoperative reductions in lateral patellar shift and tilt. Although an increase in L-PFD and a decrease in MPFD were observed after surgery, these postoperative changes did not reach the level of statistical significance. By contrast, in the OWHTO alone group, there were no significant changes in the parameters for patellar tilt and shift after surgery. In addition, although not statistically significant, an increase in L-PFD was observed after combined LR and OWHTO, while the correspond-

Table 1. Preoperative Demographics and Clinical Characteristics

\begin{tabular}{lccc}
\hline \multicolumn{1}{c}{ Variable } & OWHTO/LR group $(\mathrm{n}=19)$ & OWHTO alone group $(\mathrm{n}=18)$ & $\mathrm{p}$-value \\
\hline Age $(\mathrm{yr})$ & $52.0 \pm 9.1(32-72)$ & $63.1 \pm 7.1(53-74)$ & $\left.0.04^{\mathrm{a}}\right)$ \\
Sex $($ male), no. & 13 & 13 & $0.08^{\mathrm{b})}$ \\
Preoperative FTA $\left(^{\circ}\right)$ & $183 \pm 3.4(177-191)$ & $183 \pm 2.7(178-189)$ & 0.81 \\
Tibial slope $\left(^{\circ}\right)$ & $10.0 \pm 2.4(7-16)$ & $8.3 \pm 3.0(4-15)$ & 0.06 \\
Average opening wedge value $(\mathrm{mm})$ & $12.8 \pm 2.2(10-16)$ & $11.8 \pm 1.8(10-15)$ & 0.32 \\
Height $(\mathrm{m})$ & $1.66 \pm 0.09(1.49-1.83)$ & $1.65 \pm 0.09(1.47-1.76)$ & 0.93 \\
Weight $(\mathrm{kg})$ & $65.7 \pm 11.6(48.7-85.2)$ & $68.8 \pm 9.5(53.6-85.2)$ & 0.59 \\
BMI $\left(\mathrm{kg} / \mathrm{m}^{2}\right)$ & $23.8 \pm 3.0(19.3-29.5)$ & $25.3 \pm 3.1(24.0-30.8)$ & 0.22 \\
\hline
\end{tabular}

Values are presented as mean \pm standard deviation (range).

OWHTO: open wedge high tibial osteotomy, LR: lateral release, FTA: femorotibial angle, BMI: body mass index.

${ }^{\text {a) }}$ Statistically significant difference $(\mathrm{p}<0.05)$.

${ }^{b)}$ Fisher exact test. 
Table 2. Preoperative and Postoperative Patellar Positions in Two Groups

\begin{tabular}{|c|c|c|c|c|c|c|}
\hline \multirow{2}{*}{ Measurement } & \multicolumn{3}{|c|}{ OWHTO/LR group } & \multicolumn{3}{|c|}{ OWHTO alone group } \\
\hline & Preoperative & Postoperative & $\mathrm{p}$-value & Preoperative & Postoperative & $\mathrm{p}$-value \\
\hline Femorotibial angle $\left(^{\circ}\right)$ & $183 \pm 3.4(177-191)$ & $174 \pm 2.9(170-180)$ & $<0.01^{\text {a) }}$ & $183 \pm 2.7(178-189)$ & $172 \pm 2.0(164-193)$ & $<0.01^{\text {a) }}$ \\
\hline Tibial slope $\left(^{\circ}\right)$ & $10.0 \pm 2.4(7-16)$ & $10.3 \pm 3.0(4-18)$ & 0.99 & $8.3 \pm 3.0(4-15)$ & $7.6 \pm 4.8(2-17)$ & 0.88 \\
\hline Blackburne-Peel index & $0.82 \pm 0.2(0.5-1.2)$ & $0.69 \pm 0.1(0.5-1.0)$ & $0.03^{\text {a) }}$ & $0.77 \pm 0.2(0.4-1.5)$ & $0.58 \pm 0.2(0.2-0.9)$ & $0.04^{\mathrm{a})}$ \\
\hline Caton-Deschamps index & $0.62 \pm 0.2(0.5-1.3)$ & $0.57 \pm 0.2(0.4-1.1)$ & 0.38 & $0.95 \pm 0.2(0.5-1.2)$ & $0.77 \pm 0.2(0.3-1.0)$ & $0.03^{\mathrm{a})}$ \\
\hline Patellar lateral shift $\left({ }^{\circ}\right)$ & $11.5 \pm 3.2(5-14)$ & $9.7 \pm 4.8(1-19)$ & $0.04^{\mathrm{a})}$ & $6.8 \pm 5.2(1-20)$ & $6.4 \pm 5.2(2-17)$ & 0.35 \\
\hline Patellar lateral shift (\%) & $10.9 \pm 6.0(5.9-25.9)$ & $7.8 \pm 4.3(2.0-18.7)$ & $0.04^{\text {a) }}$ & $9.8 \pm 5.7(4.0-25.7)$ & $10.0 \pm 6.7(2.9-31.4)$ & 0.97 \\
\hline L-PFD (mm) & $8.2 \pm 2.0(5.0-12.3)$ & $9.5 \pm 4.0(3.0-20.0)$ & 0.32 & $10.4 \pm 4.8(4.0-21.3)$ & $10.2 \pm 5.0(5.0-23.0)$ & 0.94 \\
\hline M-PFD (mm) & $17.0 \pm 5.8(7.0-24.9)$ & $16.6 \pm 5.3(7.0-28.0)$ & 0.52 & $12.7 \pm 5.1(5.0-20.7)$ & $12.1 \pm 4.1(6.0-19.5)$ & 0.74 \\
\hline
\end{tabular}

Values are presented as mean \pm standard deviation (range).

OWHTO: open wedge high tibial osteotomy, LR: lateral release, L-PFD: lateral patellofemoral joint distance, M-PFD: medial patellofemoral joint distance.

${ }^{\text {a) }}$ Statistically significant difference $(\mathrm{p}<0.05)$.

ing distance was reduced after isolated OWHTO. The increase in PFD induced by concomitant LR may result in reduction of patellofemoral joint pressure.

\section{Discussion}

Although OWHTO has been reported to provide consistently satisfactory clinical outcome, the adverse effect of wedge opening proximal to the tibial tubercle on postoperative patellofemoral mechanics has been raised as a concern ${ }^{5-7,17)}$. Since arthroscopic LR has been widely utilized with the intent of improving patellofemoral mechanics and symptoms ${ }^{8}$, LR concomitant with OWHTO may potentially reduce the risk of patellofemoral complication after OWHTO.

In the present study, postoperative changes in patellar position and orientation were comparatively examined in knees undergoing OWHTO with and without concomitant LR. The comparison of pre- and postoperative radiological parameters showed postoperative reduction in patellar height in both groups. Regarding the patellar position on the axial plane as indicated by patellar shift and tilt, concomitant LR effectively reduced lateral patellar shift and tilt, while OWHTO alone did not change the alignment postoperatively.

The effect of OWHTO on postoperative patellar position and tracking has been investigated in both clinical and biomechanical studies. The majority of these studies dealt with the problem of patellar height reduction with resultant increase in patellofemoral pressure potentially leading to patellofemoral osteoarthritis ${ }^{2,418-22}$. There have been a few reports examining the change in patellar tilt and shift after OWHTO. Bito et al. ${ }^{2)}$ examined the patellofem- oral alignment after OWHTO and showed a significant decrease in lateral patellar tilt with no appreciable change in patellar shift. Based on the study results, they stated that OWHTO may negatively affect the congruency of the patellofemoral joint. Gaasbeek et al. ${ }^{20)}$ compared dynamic patellar tracking after OWHTO and closed wedge HTO by measuring three-dimensional patellar tracking in a cadaveric biomechanical study. Their study showed increased medial patellar tilt and reduced medial patellar rotation after HTO and these changes were more pronounced in OWHTO, while no significant change was observed in mediolateral patellar translation.

In the present study, both lateral patellar tilt and shift were reduced after surgery by concomitant LR at the time of OWHTO. These changes were not observed after isolated OWHTO without LR. In addition, although not statistically significant, an increase in lateral patellofemoral distance was observed after combined LR and OWHTO, while the corresponding distance was rather reduced after isolated OWHTO. In the majority of our patient population undergoing OWHTO, the patella is laterally tilted or shifted with narrowing of the lateral patellofemoral joint space. The present study results have shown decreased lateral patellar tilt and shift following combined OWHTO and LR. Therefore, it is thought that concomitant LR may effectively relieve the pressure in the lateral patellofemoral joint and potentially prevent anterior knee pain and progressive patellofemoral osteoarthritis following OWHTO especially for larger wedge size. Longino et al. ${ }^{6}$ proposed a combined tibial tubercle osteotomy with OWHTO and showed that concomitant tibial tubercle osteotomy could prevent the unfavorable patellofemoral mechanics after surgery. Although concomitant procedures to the tibial tubercle can be another op- 
tion to cope with the patellofemoral problem after OWHTO, an additional bony procedure may add to surgical invasion. LR can be concomitantly performed with minimal surgical morbidity, and the authors feel supplementation with LR can be considered especially when performing OWHTO for knees with some patellofemoral malalignment or arthritic changes.

Since each of the two involved surgeons ( $\mathrm{HN}$ and $\mathrm{SO}$ ) performed HTO with and without LR in this study, indication for LR was not specifically determined based on the study results. In addition, there are other study limitations such as small sample size, no priori power analysis performed, short follow-up period, retrospective analysis of two surgical procedures performed by two surgeons ( $\mathrm{HN}$ and $\mathrm{SO}$ ), no randomization for the two procedures (a concern for selection bias), and the lack of clinical results. A prospective comparative study including clinical evaluation for a larger number of patients with a longer follow-up period would be required to critically examine the effect of concomitant LR on the surgical outcome of OWHTO.

\section{Conclusions}

OWHTO performed for varus osteoarthritis knees induced a postoperative decrease in patellar height in both patient groups with and without concomitant LR. Regarding the change in patellofemoral alignment, however, concomitant LR in OWHTO significantly decreased lateral patellar tilt and shift, while no significant difference in those parameters was noted in the HTO alone knees.

\section{Conflict of Interest}

No potential conflict of interest relevant to this article was reported.

\section{References}

1. Backstein D, Meisami B, Gross AE. Patella baja after the modified Coventry-Maquet high tibial osteotomy. J Knee Surg. 2003;16:203-8.

2. Bito H, Takeuchi R, Kumagai K, Aratake M, Saito I, Hayashi R, Sasaki Y, Saito T. Opening wedge high tibial osteotomy affects both the lateral patellar tilt and patellar height. Knee Surg Sports Traumatol Arthrosc. 2010;18:955-60.

3. Bode G, Ogon P, Pestka J, Zwingmann J, Feucht M, Sudkamp N, Niemeyer P. Clinical outcome and return to work following single-stage combined autologous chondrocyte implan- tation and high tibial osteotomy. Int Orthop. 2015;39:68996.

4. Brouwer RW, Bierma-Zeinstra SM, van Koeveringe AJ, Verhaar JA. Patellar height and the inclination of the tibial plateau after high tibial osteotomy: the open versus the closedwedge technique. J Bone Joint Surg Br. 2005;87:1227-32.

5. Chae DJ, Shetty GM, Wang KH, Montalban AS Jr, Kim JI, Nha KW. Early complications of medial opening wedge high tibial osteotomy using autologous tricortical iliac bone graft and T-plate fixation. Knee. 2011;18:278-84.

6. Longino PD, Birmingham TB, Schultz WJ, Moyer RF, Giffin JR. Combined tibial tubercle osteotomy with medial opening wedge high tibial osteotomy minimizes changes in patellar height: a prospective cohort study with historical controls. Am J Sports Med. 2013;41:2849-57.

7. Matthews LS, Goldstein SA, Malvitz TA, Katz BP, Kaufer H. Proximal tibial osteotomy. Factors that influence the duration of satisfactory function. Clin Orthop Relat Res. 1988; (229):193-200.

8. Ogilvie-Harris DJ, Jackson RW. The arthroscopic treatment of chondromalacia patellae. J Bone Joint Surg Br. 1984;66: 660-5.

9. Christodoulou NA, Tsaknis RN, Sdrenias CV, Galanis KG, Mavrogenis AF. Improvement of proximal tibial osteotomy results by lateral retinacular release. Clin Orthop Relat Res. 2005;441:340-5.

10. El-Azab H, Glabgly P, Paul J, Imhoff AB, Hinterwimmer S. Patellar height and posterior tibial slope after open- and closed-wedge high tibial osteotomy: a radiological study on 100 patients. Am J Sports Med. 2010;38:323-9.

11. Kaper BP, Bourne RB, Rorabeck CH, Macdonald SJ. Patellar infera after high tibial osteotomy. J Arthroplasty. 2001;16: 168-73.

12. Stoffel K, Willers C, Korshid O, Kuster M. Patellofemoral contact pressure following high tibial osteotomy: a cadaveric study. Knee Surg Sports Traumatol Arthrosc. 2007;15:1094100.

13. Saito T, Takeuchi R, Ara Y, Yoshida T, Koshino T. High tibial osteotomy with anterior advancement of distal fragment for medial and patellofemoral compartmental osteoarthritis of the knee. Knee. 2002;9:127-32.

14. Ford DH, Post WR. Open or arthroscopic lateral release. Indications, techniques, and rehabilitation. Clin Sports Med. 1997;16:29-49.

15. Elkousy H. Complications in brief: arthroscopic lateral release. Clin Orthop Relat Res. 2012;470:2949-53. 
16. El-Azab H, Halawa A, Anetzberger H, Imhoff AB, Hinterwimmer S. The effect of closed- and open-wedge high tibial osteotomy on tibial slope: a retrospective radiological review of 120 cases. J Bone Joint Surg Br. 2008;90:1193-7.

17. Gaasbeek RD, Sonneveld H, van Heerwaarden RJ, Jacobs WC, Wymenga AB. Distal tuberosity osteotomy in open wedge high tibial osteotomy can prevent patella infera: a new technique. Knee. 2004;11:457-61.

18. Phillips CL, Silver DA, Schranz PJ, Mandalia V. The measurement of patellar height: a review of the methods of imaging. J Bone Joint Surg Br. 2010;92:1045-53.

19. Flahiff CM, Kraus VB, Huebner JL, Setton LA. Cartilage mechanics in the guinea pig model of osteoarthritis studied with an osmotic loading method. Osteoarthritis Cartilage. 2004;12:383-8.

20. Gaasbeek R, Welsing R, Barink M, Verdonschot N, van Kampen A. The influence of open and closed high tibial osteotomy on dynamic patellar tracking: a biomechanical study. Knee Surg Sports Traumatol Arthrosc. 2007;15:97884.

21. Fan JC. Open wedge high tibial osteotomy: cause of patellar descent. J Orthop Surg Res. 2012;7:3.

22. Agarwala S, Sobti A, Agrawal P. A report of nonunion at medial wedge high tibial osteotomy site and its management. J Nat Sci Biol Med. 2015;6(Suppl 1):S160-2. 